\title{
@creative
}

\section{A decomposition for Markov processes at an independent exponential time}

\author{
Mihael Perman * \\ Faculty of Mathematics, Natural Sciences and Information Technologies, \\ University of Primorska, Glagoljaška 8, 6000 Koper, Slovenia \\ and \\ Department of Mathematics, University of Ljubljana, \\ Jadranska 19, 1000 Ljubljana, Slovenia
}

Received 29 September 2015, accepted 12 October 2015, published online 22 April 2016

\begin{abstract}
The path of Markov process $X$ run up to an independent exponential random time $S_{\theta}$ can be decomposed into the part prior to the last exit time from a point before $S_{\theta}$, and the remainder up to $S_{\theta}$. In this paper the laws of the two segments are identified under suitable assumptions using excursion theory.
\end{abstract}

Keywords: Markov processes, excursions, last exit decomposition, diffusions, Brownian motion.

Math. Subj. Class.: 60J25, 60J55, 60J60, 60J65, 60G51.

\section{Introduction}

Considering a Markov process $X$ up to an independent exponential time $S_{\theta}$ with rate $\theta>0$ has been used effectively to compute functionals of $X$. The computations are often based on decompositions of the path of $X$ up to $S_{\theta}$ into fragments before and after the last exit time from a set before time $S_{\theta}$. The known results described below are more general in the sense that the path is decomposed at the last exit from a set before either fixed times or random times belonging to a suitable family. Choosing an independent exponential time in some cases leads to simpler descriptions of the laws of the two fragments involved. They are often conditionally independent given suitable conditioning variables and their laws are related to laws of known processes.

Williams [27] uses a decomposition of Brownian motion with drift run up to an independent $S_{\theta}$ to prove result of Ray [23] on the distribution of local times in the space

\footnotetext{
*This work is supported in part by the Slovenian Research Agency (research program P1-0285).
}

E-mail address: mihael.perman@fmf.uni-lj.si (Mihael Perman) 
variable. In their investigations of Ray-Knight theorems for Brownian motion $B$ at fixed times Biane and Yor [4] considered the pair of processes $\left(B_{t}: 0 \leq g_{S_{\theta}}\right)$ and $\left(B_{S_{\theta}-t}: 0 \leq\right.$ $\left.t \leq S_{\theta}-g_{S_{\theta}}\right)$ where $S_{\theta}$ is an exponential random variable independent of $B$ and $g_{t}=$ $\sup \left\{s \leq t: B_{s}=0\right\}$ is the last exit time from 0 before time $t$. Under $P_{0}$ the two processes are shown to be independent and their conditional laws given the local time $L\left(S_{\theta}\right)$ at zero of Brownian motion and $B_{S_{\theta}}$ respectively are identified. This decomposition has been exploited by Jeanblanc, Pitman and Yor [13] to derive Feynman-Kac formulae for Brownian motion. Salminen, Vallois and Yor [26] extend the decomposition for Brownian motion to linear diffusions on $[0, \infty)$ with 0 a recurrent point and use them to study the excursion of the diffusion straddling an independent exponential time.

For general Markov processes Pittenger and Shih [22] investigated the dependence of the fragments of the path of a càdlàg strong Markov process $X$ before coterminal time $L^{t} \leq t$ and the fragment on the interval between $L^{t}$ and $t$. Last exit times $L_{F}^{t}$ before time $t$ from a closed set $F$ are coterminal times. It is shown that given a suitably defined $\sigma$-algebra $\mathcal{F}_{L^{t}}$, the conditional law of the process $\left(X_{L_{t}+s}: 0 \leq s \leq t-L^{t}\right)$ only depends on $L_{t}$ and $X_{L_{t}^{F}}$ or $X_{L^{t}-}$ and is an inhomogenous strong Markov process. Getoor and Sharpe [7] give related results. General and elegant treatments of last exit decompositions are given in Maisonneuve [18] and Pitman [21]. Kallenberg [14] proves that for Lévy processes the fragments considered by Pittenger and Shih are conditionally independent given $X_{L_{F}^{t}-}$ and $L_{F}^{t}$ where the last exit time from a set $F$ is an instance of a backward time. Under suitable conditions the laws of the two fragments are described.

Another example of considering a decomposition of a Markov process at last exit time from 0 before and independent exponential time $S_{\theta}$ is the proof of fluctuation equalities for Lévy processes given by Greenwood and Pitman [9]. If $X$ is a Lévy process then it is known that the process reflected at the supremum defined by $Y_{t}=\sup _{s \leq t} X_{s}-X_{t}$ is a strong Markov process. See e.g. Bertoin [2], p.156. If $Y$ is split at the last exit time from 0 before an independent exponential time $S_{\theta}$ the two fragments are independent and their laws can be described. This gives a direct proof of the infinite divisibility results needed to prove the fluctuation identities by Pečerskiı̌ and Rogozin [20].

The setting of this paper is a strong Markov processes $X$ with a recurrent point $a$. Last exit times from $a$ are considered and the path of $X$ is split at the last exit time from $a$ before an independent exponential time $S_{\theta}$. The two resulting fragments turn out to be independent and their laws are described.

\section{Notation and statement}

Let $X$ be a càdlàg Markov process with state space $(E, \mathcal{E})$ which we will assume to be Lusinian. We will assume that the semigroup $P_{t}$ maps Borel functions into Borel functions and that the process can be realized as the coordinate process on the Skorohod space $\Omega$ of paths which are right continuous with left limits. Assume that the Markov process has transition densities $p_{t}(x, y)$ with respect to a $\sigma$-finite measure $\xi$ on $(E, \mathcal{E})$. The densities are assumed to be jointly continuous in all three variables for $t>0$ which implies the Chapman-Kolmogorov equations

$$
p_{t+s}(x, y)=\int_{E} p_{t}(x, z) p_{s}(z, y) \xi(d z)
$$

for all $s, t>0$ and $x, y \in E$. 
To formulate the results the existence of a dual strong Markov process $\hat{X}$ on $(E, \mathcal{E})$ relative to the measure $\xi$ will be assumed. This means that $\xi$ is an invariant measure for both $X$ and $\hat{X}$ and

$$
P_{x}\left(X_{t} \in d y\right)=p_{t}(x, y) \xi(d y) \quad \text { and } \quad \hat{P}_{x}\left(\hat{X}_{t} \in d y\right)=\hat{p}_{t}(y, x) \xi(d y)
$$

for all $t>0, x, y \in E$ with $\hat{p}_{t}(x, y)=p_{t}(y, x)$. See [6] and the references therein for details. Denote by

$$
r_{\theta}(x, y)=\int_{0}^{\infty} e^{-\theta t} p_{t}(x, y) d t \quad \text { and } \quad \hat{r}_{\theta}(x, y)=\int_{0}^{\infty} e^{-\theta t} \hat{p}_{t}(x, y) d t
$$

the resolvent densities of $X$ and $\hat{X}$ respectively. For the sake of simplicity it will be assumed that $X$ and $\hat{X}$ have infinite lifetimes $\zeta$ under $P_{x}$ and $\hat{P}_{y}$ for all $x, y \in E$ respectively.

The assumptions on $X$ imply that it is possible to define bridge laws

$$
P_{x, y}^{t}(\cdot)=P_{x}\left(\cdot \mid X_{t}=y\right)
$$

for $t>0$ and for $x, y \in E$. By Proposition 1 in Fitzsimmons, Pitman, Yor [6] for any $x, y \in E$ and $t>0$ with $p_{t}(x, y)>0$ there is a unique law $P_{x, y}^{t}$ on $\left(\Omega, \mathcal{F}_{t}\right)$ such that for any $\mathcal{F}_{s}$-measurable functional $F$ for $0 \leq s<t$

$$
E_{x, y}^{t}(F) \cdot p_{t}(x, y)=E_{x}\left(F \cdot p_{t-s}\left(X_{s}, y\right)\right)
$$

where $E_{x, y}^{t}$ and $E_{x}$ are expectations with respect to measures $P_{x, y}^{t}$ and $P_{x}$ respectively. The laws $P_{x, y}^{t}$ provide a regular version of the family of conditional distributions $P\left(\cdot \mid X_{t}=y\right)$. Furthermore by Corollary 1 in Fitzsimmons, Pitman, Yor [6] the law of the reversed bridge $\left(X_{(t-s)-}: 0 \leq s<t\right)$ under $P_{x, y}^{t}$ has the law of the bridge of the dual process $\hat{P}_{y, x}^{t}$.

The subject of this paper is the law of the process $X$ started at $a$ and run to an independent exponential time $S_{\theta}$ with rate $\theta$ and conditioned on $\left\{X_{S_{\theta}}=b\right\}$. Conditionally on $\left\{S_{\theta}=t, X_{t}=b\right\}$ the law of the process will be the law of the bridge $P_{a, b}^{t}$ and the laws $P_{a, b}^{t}$ will serve as the regular version of the family of conditional distributions.

Assume that $a$ is a recurrent point of the process $X$. Let $T_{a}=\inf \left\{t>0: X_{t-}=\right.$ $a$ or $\left.X_{t}=a\right\}$. Since $a$ is assumed to be recurrent the assumptions imply that $P_{b}\left(T_{a}<\right.$ $\infty)=1$ for all $b \in E$.

For $t>0$ define the last exit time from $a$ before time $t$ as

$$
g_{t}=\sup \left\{s \leq t: X_{t-}=a \text { or } X_{t}=a\right\} .
$$

Let $\left(L_{t}^{a}: t \geq 0\right)$ be the local time for the process $X$ at $a$. We will assume that such a right continuous nondecreasing additive functional exists and only increases on the set $M=\left\{t \geq 0: X_{t-}=a\right.$ or $\left.X_{t}=a\right\}$. All the results will be valid for any choice of normalization of the local time. Let for $s \geq 0$

$$
\tau_{s}=\inf \left\{t \geq 0: L_{t}^{a}>s\right\}
$$

be the right continuous inverse of the local time. From the strong Markov property of $X$ it follows that $\left(\tau_{s}: s \geq 0\right)$ is a subordinator. Since we are assuming recurrence the local time at $a$ will be unbounded and hence $\tau_{s}$ is well defined for all $s$. For simplicity we will assume that the set $M$ has Lebesgue measure 0 almost surely. This means that the subordinator 
$\left(\tau_{s}: s \geq 0\right)$ has no drift. From properties of subordinators, see Bertoin [2], Ch. 3, it follows that

$$
E_{a}\left(e^{-\theta \tau_{u}}\right)=e^{-\psi(\theta) u} .
$$

The notation $X(t), L^{a}(t)$ and $g(t)$ will be used for $X_{t}, L_{t}^{a}$ and $g_{t}$ whenever necessary. The theorem to be proved is stated as follows.

Theorem 2.1. Assume that a is a recurrent point for the process $X$ and $p_{t}(a, b)>0$ for all $t>0$. Let $X_{0}=a$ and assume $\left(L_{t}^{a}: t \geq 0\right)$ is the local time of $X$ at $a$. If $S_{\theta}$ is an exponential random variable with parameter $\theta$ independent of $X$ then, under the measure $P_{a}$ :

(i) The random variables $L^{a}\left(S_{\theta}\right)$ and $X\left(S_{\theta}\right)$ are independent with distributions

$$
P_{a}\left(L^{a}\left(S_{\theta}\right) \in d u\right)=\psi(\theta) e^{-\psi(\theta) u} d u \quad \text { and } \quad P_{a}\left(X\left(S_{\theta}\right) \in d y\right)=\theta r_{\theta}(a, y) d y
$$

where $\psi(\theta)$ is the Laplace exponent defined in Equation 2.5.

(ii) The processes

$$
\left(X_{t}: 0 \leq t \leq g_{S_{\theta}}\right) \quad \text { and } \quad\left(X_{g_{S_{\theta}}+u}: 0 \leq u \leq S_{\theta}-g_{S_{\theta}}\right)
$$

are independent.

(iii) For bounded measurable functionals $F$ and $G$

$$
\begin{gathered}
E_{a}\left[F\left(X_{s}: 0 \leq s \leq g_{S_{\theta}}\right) \mid L_{S_{\theta}}=u\right]=\frac{E_{a}\left[F\left(X_{s}: 0 \leq s \leq \tau_{u}\right) e^{-\theta \tau_{u}}\right]}{E_{a}\left[e^{-\theta \tau_{u}}\right]} \\
E_{a}\left[G\left(X_{\left(S_{\theta}-s\right)-}: 0 \leq s \leq S_{\theta}-g_{S_{\theta}}\right) \mid X_{S_{\theta}}=b\right]= \\
=\frac{\hat{E}_{b}\left[G\left(\hat{X}_{s}: 0 \leq s \leq \hat{T}_{a}\right) e^{-\theta \hat{T}_{a}}\right]}{\hat{E}_{b}\left[e^{-\theta \hat{T}_{a}}\right]}
\end{gathered}
$$

where $\hat{E}_{b}$ refers to expectation under the law $\hat{P}_{b}$ of the dual process, and $\hat{T}_{a}=\inf \{t$ : $\hat{X}_{t}=$ a or $\left.\hat{X}_{t-}=a\right\}$ is the hitting time of a for $\hat{X}$.

The novelty lies in the fact that known special cases are covered by the more general Markov setup. Excursion arguments used are standard.

\section{Excursion arguments}

Let $\Pi$ be a point process on an abstract space $(S, \mathcal{S})$ with mean measure $\Lambda$. If $\Pi$ is a Poisson process then by Campbell's Theorem, see Kingman [16], p. 28, for any measurable $f \geq 0$

$$
E\left(\exp \left(-\int_{S} f(x) \Pi(d x)\right)\right)=\exp \left(-\int_{S}\left(1-e^{-f(x)}\right) \Lambda(d x)\right) .
$$

Conversely, if Equation 3.1 holds for any measurable $f \geq 0$, then $\Pi$ is a Poisson process with mean measure $\Lambda$. 
Assume that $\Pi$ is a Poisson process with mean measure $\Lambda$, and let $h: S \rightarrow[0, \infty)$ be a measurable function such that

$$
\int_{S}\left(1-e^{-h(x)}\right) \Lambda(d x)<\infty .
$$

The random variable $\Sigma_{h}$ defined by

$$
\Sigma_{h}=\int_{S} h(x) \Pi(d x)
$$

is almost surely finite and non-negative with

$$
E\left(\exp \left(-\Sigma_{h}\right)\right)=\exp \left(-\int_{S}\left(1-e^{-h(x)}\right) \Lambda(d x)\right) .
$$

Define a new probability measure $Q$ by

$$
\frac{d Q}{d P}=\frac{\exp \left(-\Sigma_{h}\right)}{E\left[\exp \left(-\Sigma_{h}\right)\right]} .
$$

The following lemma is known in the literature, see Proposition 2.1 in James [12] and the discussion therein, or Proposition 2.4 in Bertoin [3].

Lemma 3.1. Under the measure $Q, \Pi$ is a Poisson process with mean measure $e^{-h(x)}$. $\Lambda(d x)$.

Proof. It suffices to check that Equation 3.1 holds. Denote $c=1 / E\left(\exp \left(-\Sigma_{h}\right)\right)$ and let $\Sigma_{f}=\int_{S} f(x) \Pi(d x)$ for a measurable funtion $f \geq 0$. One has

$$
\begin{aligned}
E_{Q}\left[\exp \left(-\Sigma_{f}\right)\right] & =c E_{P}\left[\exp \left(-\Sigma_{h}\right) \cdot \exp \left(-\int_{S} f(x) \Pi(d x)\right)\right] \\
& =c E_{P}\left[\exp \left(-\int_{S}(f(x)+h(x)) \Pi(d x)\right)\right] \\
& =c \exp \left(-\int_{S}\left(1-e^{-f(x)-h(x)}\right) \Lambda(d x)\right) \\
& =c \exp \left(-\int_{S}\left[\left(1-e^{-f(x)}\right) e^{-h(x)}+\left(1-e^{-h(x)}\right)\right] \Lambda(d x)\right) \\
& =\exp \left(-\int_{S}\left(1-e^{-f(x)}\right) e^{-h(x)} \Lambda(d x)\right)
\end{aligned}
$$

Let $X$ be a càdlàg strong Markov process. The set $M=\left\{t \geq 0: X_{t-}=a\right.$ or $\quad X_{t}=$ $a\}$ is closed under the assumptions. Since we are assuming recurrence of $X$ the complement of $M$ is a countable union of bounded open intervals. The segments of the path of $X$ on these open intervals are called the excursions of $X$ away from the point $a$. By definition the open intervals coincide with the complement of the range of the subordinator $\left(\tau_{s}: s>0\right)$ defined in Equation 2.4. Let $U_{\delta}$ be the space of càdlàg paths 
$w:[0, \infty) \rightarrow E$ such that $w(0)=a$ and there is a $t>0$ such that for $0<s<t$ we have $w(s) \neq a, w(s-) \neq a$, and $w(t)=a$ or $w(t-)=a$ and $w(s)=\delta$ for $s>t$ where $\delta$ is the coffin state added to $E$. Let $\mathcal{U}_{\delta}$ be the $\sigma$-algebra generated by the coordinate maps in $U_{\delta}$. Define the point process $\left(e_{s}: s>0\right)$ of excursions of $X$ in the sense of Itô as

$$
e_{s}= \begin{cases}\delta & \text { if } \tau_{s}-\tau_{s-}=0 \\ e_{s}(u)=X_{\tau_{s-}+u} & \text { for } u<\tau_{s}-\tau_{s-} \text { and } \delta \text { else }\end{cases}
$$

The process $e$ is a Poisson process in the sense of Itô governed by the measure $\tilde{\lambda} \times n$ where $\tilde{\lambda}$ is a multiple of the Lebesgue measure on $[0, \infty)$ and $n$ is the Itô excursion law. We can change the normalization of the local time, if needed, in order to ensure that $\tilde{\lambda}$ is the standard Lebesgue measure. See Rogers and Williams [25], Ch. 8 or Revuz and Yor [24], Ch. 8 for background on excursion theory.

The connection between excursion theory and the law of a Markov process run up to an independent exponential time $S_{\theta}$ is established through marking excursions. Let $\Pi$ be a Poisson process on an abstract space $(S, \mathcal{S})$. If conditionally on $\Pi$ each point $x \in \Pi$ is assigned a mark with probability $p(x)$ independently of all the other points in $\Pi$ then the resulting marked and unmarked processes are both Poisson and are independent. If $\Lambda$ is the mean measure of $\Pi$ the marked process will have mean measure $p \cdot \Lambda$ and the unmarked process $(1-p) \cdot \Lambda$. See Kingman [16], Ch. 5 for definitions and proofs.

The excursion of the Markov process $X$ straddling the independent exponential random time $S_{\theta}$ can be interpreted as the first marked excursion of $X$ where conditionally on $e$ marks are assigned to an excursion $e$ with probability $1-e^{-\theta R(e)}$ where $R(e)$ stands for the duration of the excursion i.e. the length of the open interval of $M^{c}$ containing $S_{\theta}$. Intuitively we can think that $S_{\theta}$ is the first point in a Poisson process $N$ on $(0, \infty)$ with rate $\theta$ and independent of $X$. Excursions straddling a point of $N$ are considered marked and other excursions are considered unmarked. By independence properties of Poisson processes conditionally on $e$ the marks are assigned independently and an excursion of length $R(e)$ contains a point of the Poisson process with probability $1-e^{R(e)}$ which follows from the Poisson distribution of points contained in the excursion interval. See Sec. 49 in Rogers and Williams [25] for definitions and proofs.

The following theorem is stated in slightly more general terms allowing the Poisson process $\left(e_{s}: s>0\right)$ to be killed at a rate $q$. This would correspond to excursions of $X$ that have infinte length. The conclusions of the theorem are well known, see Sec. 49.4 in Rogers and Williams [25].

Theorem 3.2. Let $\left(e_{s}: s>0\right)$ be a possibly killed excursion process of $X$ from a recurrent point $a$ in the sense of Itô, and let $S_{\theta}$ be an exponential random variable with parameter $\theta$ independent of $X$. Denote by $\left(L_{t}^{a}: t \geq 0\right)$ the local time process of $X$ at a and $\left(e_{s}: s>0\right)$ the associated excursion process governed by $\lambda \times n$ where $\lambda$ is the Lebesgue measure.

(i) The local time $L^{a}\left(S_{\theta}\right)$ during the excursion straddling $S_{\theta}$ is an exponential random variable with parameter

$$
\int_{U}\left(1-e^{-\theta R(e)}\right) n(d e ; R<\infty)+q
$$

where $q$ is the rate of arrival of excursions with infinite lifetime. Moreover, it is independent of the excursion $e^{*}=e\left(L_{S_{\theta}}^{a}\right)$ which may have infinite lifetime. 
(ii) Given $L^{a}\left(g_{S_{\theta}}\right)=u$ the process of excursions $\left(e_{s}: 0<s<u\right)$ is a Poisson process in the sense of Itô which is governed by the measure $\bar{n}$ given by $\bar{n}(d e)=$ $e^{-\theta R(e)} n(d e ; R(e)<\infty)$ where $n$ is Itô's excursion law and $R(e)$ denotes the length of the excursion. Moreover, $e^{*}$ is conditionally independent of $\left(e_{s}: 0<s<u\right)$ given $\left\{L^{a}\left(S_{\theta}\right)=u\right\}$, and is independent of $L^{a}\left(S_{\theta}\right)$.

Proof. The first marked excursion in $\left(e_{s}: s>0\right)$ will arrive at an exponential time. The processes of finite length excursions and those of infinte length are independent so the rates of arrivals add. The rate of arrivals of marked finite length excursions is by definition equal to

$$
\int_{U}\left(1-e^{-\theta R(e)}\right) n(d e ; R<\infty)
$$

The two processes of marked and unmarked excursions are independent. This means that conditionally on $L^{a}\left(S_{\theta}\right)=u$ the process $\left(e_{s}: 0<s<u\right)$ is a Poisson process on $(0, u) \times U_{\delta}$.

The first marked excursion is picked according to the normalized law $\left(1-e^{-\theta R}\right) \cdot n$ irrespective of the local time $L^{a}\left(S_{\theta}\right)$. This and the independence of marked and unmarked excursions conclude the proof.

\section{Proofs}

Recall that under the assumptions on $X$ and $\hat{X}$ and if $p_{t}(a, b)>0$ there is a measure $P_{a, b}^{t}$ corresponding to the bridge of $X$ starting at $a$ and conditioned to be $b$ at time $t$. The family of $P_{a, b}^{t}$ is a family of regular conditional laws of $X$ given $X_{t}=b$. If $S_{\theta}$ is an exponential random variable of rate $\theta$ then under $P_{a}$ the family $P_{a, b}^{t}$ is a regular conditional law of $X$ given $\left\{S_{\theta}=t, X_{t}=b\right\}$. The assumptions made on $X$ and $\hat{X}$ also imply that $X$ and $\hat{X}$ have no jumps at fixed times. See (3.18) in Getoor and Sharpe [8] for a proof. Let $\hat{T}_{b}=\inf \left\{t>0: \hat{X}_{t}=b\right.$ or $\left.\hat{X}_{t-}=b\right\}$. Assume further that $\hat{P}_{b}\left(\hat{T}_{b}>0\right)=0$ which in conjunction with right continuity and strong Markov property implies that $X_{T_{b}}=b$.

Lemma 4.1. Assume that $P_{a}\left(T_{b}<\infty\right)=1$. Assume that $p_{t}(a, b)>0$ for all $t>0$. Then

$$
P_{a}\left(T_{b} \in d s \mid X\left(S_{\theta}\right)=b\right)=e^{-\theta s} P_{a}\left(T_{b} \in d s\right) \frac{r_{\theta}(b, b)}{r_{\theta}(a, b)},
$$

where $r_{\theta}(x, y)$ is the resolvent density.

Proof. By assumptions on $b$ and $\hat{X}$ we have $P_{a}\left(T_{b}<S_{\theta} \mid X\left(S_{\theta}\right)=b\right)=1$. For fixed $0<s<t$ and a $\mathcal{F}_{s}$ measurable functional $F$ by 2.6 in Fitzsimmons, Pitman and Yor [6]

$$
E_{a, b}^{t}(F) \cdot p_{t}(a, b)=E_{a}\left(F \cdot p_{t-s}\left(X_{s}, b\right)\right)
$$

Choose an open neighbourhood $B \in \mathcal{E}$ of $b$ such that $r_{\theta}(a, y)>0$ for $y \in B$. Let 
$F=1\left(T_{b} \in d s\right)$. We have

$$
\begin{aligned}
P_{a} & \left(T_{b} \in d s, T_{b}<S_{\theta}, X_{S_{\theta}} \in B\right)= \\
& =\int_{s}^{\infty} \theta e^{-\theta t} d t \int_{B} p_{t}(a, y) P_{a, y}^{t}\left(T_{b} \in d s\right) \xi(d y) \\
& =\int_{s}^{\infty} \theta e^{-\theta t} d t \int_{B} P_{a}\left(T_{b} \in d s\right) p_{t-s}(b, y) \xi(d y) \\
& =e^{-\theta s} P_{a}\left(T_{b} \in d s\right) \int_{B} \xi(d y) \int_{0}^{\infty} \theta e^{-\theta u} p_{u}(b, y) d u \\
& =e^{-\theta s} P_{a}\left(T_{b} \in d s\right) \theta \int_{B} r_{\theta}(b, y) \xi(d y) \\
& =e^{-\theta s} P_{a}\left(T_{b} \in d s\right) \int_{B} \frac{r_{\theta}(b, y)}{r_{\theta}(a, y)} \cdot \theta r_{\theta}(a, y) \xi(d y)
\end{aligned}
$$

It follows that

$$
P_{a}\left(T_{b} \in d s \mid X\left(S_{\theta}\right)=b\right)=e^{-\theta s} P_{a}\left(T_{b} \in d u\right) \frac{r_{\theta}(b, b)}{r_{\theta}(a, b)} .
$$

Remark 4.2. Integrating the equation with respect to $s$ over $(0, \infty)$ in Lemma 4.1 gives the well known formula

$$
E_{a}\left(e^{-\theta T_{b}}\right)=\frac{r_{\theta}(a, b)}{r_{\theta}(b, b)}
$$

See e.g. Rogers and Williams [25], (50.7) on p. 293.

Let us consider the process $\left(X_{t}: 0 \leq t \leq T_{b}\right)$ given $\left\{X_{S_{\theta}}=b\right\}$. The following lemma gives the conditional distribution of this process given $X\left(S_{\theta}\right)=b$.

Lemma 4.3. Assume that $P_{a}\left(T_{b}<\infty\right)=1$. Assume that $p_{t}(a, b)>0$ for all $t>0$. Let $F$ be a non-negative measurable functional of the process $\left(X_{t}: 0 \leq t \leq T_{b}\right)$. Then

$$
E_{a}\left(F \mid X\left(S_{\theta}\right)=b\right)=E_{a}\left(e^{-\theta T_{b}} \cdot F\right) \cdot \frac{r_{\theta}(b, b)}{r_{\theta}(a, b)},
$$

where $r_{\theta}(x, y)$ is the resolvent density.

Proof. As in Lemma 4.1 we compute for an open neighbourhood $B \in \mathcal{E}$ of $b$ such that 
$r_{\theta}(a, y)>0$ and a bounded measurable functional $F$

$$
\begin{aligned}
E_{a} & {\left[F \cdot 1\left(T_{b}<S_{\theta}, T_{b} \in d s, X_{S_{\theta}} \in B\right)\right]=} \\
= & \int_{s}^{\infty} \theta e^{-\theta t} d t \int_{B} p_{t}(a, y) E_{a, y}^{t}\left(F \cdot 1\left(T_{b} \in d s\right)\right) d y \\
& =\int_{s}^{\infty} \theta e^{-\theta t} d t \int_{B} E_{a}\left(F \cdot 1\left(T_{b} \in d s\right)\right) p_{t-s}(b, y) d y \\
& =e^{-\theta s} E_{a}\left(F \cdot 1\left(T_{b} \in d s\right)\right) \int_{B} d y \int_{0}^{\infty} \theta e^{-\theta u} p_{u}(b, y) d u \\
& =e^{-\theta s} E_{a}\left(F \cdot 1\left(T_{b} \in d s\right)\right) \theta \int_{B} r_{\theta}(b, y) d y \\
& =e^{-\theta s} E_{a}\left(F \cdot 1\left(T_{b} \in d s\right)\right) \int_{B} \frac{r_{\theta}(b, y)}{r_{\theta}(a, y)} \cdot \theta r_{\theta}(a, y) d y
\end{aligned}
$$

This in conjunction with the distribution of $T_{b}$ from Lemma 4.1 completes the proof.

The conclusions of Lemma 4.3 apply equally to the dual process $\hat{X}$. Moreover under $P_{a}$ the conditional law of the process $\left(X_{\left(S_{\theta}-t\right)-}: 0 \leq t \leq S_{\theta}\right)$ given $X_{S_{\theta}}=b$ is equal to the law of $\hat{X}$ started at $b$ run to an independent exponential time and conditioned to be $a$ at the end. This implies that under the assumptions on $a$ for any bounded functional $G$

$$
\begin{aligned}
E_{a}\left[G\left(X_{\left(S_{\theta}-t\right)-}: 0 \leq t \leq S_{\theta}-g_{S_{\theta}}\right) \mid X_{S_{\theta}}=b\right] \\
=\hat{E}_{b}\left[G\left(\hat{X}_{t}: 0 \leq t \leq \hat{T}_{a}\right) e^{-\theta \hat{T}_{a}}\right] \cdot \frac{\hat{r}_{\theta}(a, a)}{\hat{r}_{\theta}(b, a)}
\end{aligned}
$$

because the last exit time $g_{S_{\theta}}$ from $a$ is the first hitting time of $a$ for the reversed process. This is in accordance with Theorem 7.6 in Getoor and Sharpe [8] that excursions straddling a fixed time reversed and conditioned on the length are the excursions of the dual process. See also formula (3.12) in Ikeda, Nagasawa, Sato [11] who give the law of the process reversed from the lifetime of a killed Markov process. The case treated here considers killing at a constant rate.

We are now in position to give the proof of Theorem 2.1.

Proof. The second assertion in (i) is the definition of the resolvent density. Let $\left(e_{s}: s>0\right)$ be the excursion process of $X$ from $a$. Marked excursions arrive at an exponential rate so we know that $L^{a}\left(S_{\theta}\right)$ will be exponential. Since excursions are marked by an independent Poisson process, the event $\left\{L^{a}\left(S_{\theta}\right)>u\right\}$ is equal to the event that there is no mark in the interval $\left[0, \tau_{u}\right]$, and has conditional probability $e^{-\theta \tau_{u}}$. Integration gives

$$
P_{a}\left(L^{a}\left(S_{\theta}\right)>u\right)=E_{a}\left(e^{-\theta \tau_{u}}\right)=e^{-u \psi(\theta)},
$$

which by differentiation gives the density. To prove (ii) note that by Theorem 3.2 conditionally on $\left\{L^{a}\left(S_{\theta}\right)=u\right\}$ the process of excursions $\left(e_{s}: 0<s<u\right)$ is independent of the excursion $e^{*}$ straddling $S_{\theta}$. Because marks to excursions are assigned by an independent Poisson process conditionally on $R\left(e^{*}\right)=r$ the mark is distributed at the distance $U$ from the left endpoint with density

$$
\theta e^{-\theta u} /\left(1-e^{-\theta r}\right)
$$


on $[0, r]$ independently of the process of unmarked excursions and of the local time $L^{a}\left(S_{\theta}\right)$. So the excursion $e^{*}$ together with the position of $S_{\theta}$ within the duration of $e^{*}$ are independent of the process of unmarked excursions and of $L^{a}\left(S_{\theta}\right)$. This proves the independence of the two processes in (ii).

For the first assertion in (iii) note that conditionally on $L^{a}\left(S_{\theta}\right)=u$ the excursions of $\left(X_{t}: 0 \leq t \leq g_{S_{\theta}}\right)$ from the point $a$ are a Poisson process with excursion law $e^{-\theta R} \cdot n$ by Theorem 3.2 (i). On the other hand, if we let $\left(e_{s}: 0<s<u\right)$ be the Poisson process of excursions of $X$ from $a$ and choose $h(e)=\theta R(e)$ in Lemma 3.1, under the new measure the process is still Poisson but with the mean measure $e^{-\Sigma_{h}} \cdot n$. But under the assumption that the set $M$ has Lebesgue measure 0 we have $\Sigma_{h}=\theta \cdot \tau_{u}$.

The proof of the second formula in (iii) follows from Lemma 4.3 applied to the reversed process.

Note the connection with Lemma 4.1 in Kallenberg [14] which states that for Lévy processes with continuous densities

$$
P_{a}\left(F\left(X_{s}: 0 \leq s \leq \tau_{u}\right) \mid \tau_{u}=t\right)=P_{a, a}^{t}\left(F\left(X_{s}: 0 \leq s \leq t\right) \mid L_{t}=u\right)
$$

where $P_{t}$ refers to the law of the bridge of length $t$. Noting that Lemma 3.1 gives

$$
P_{a}\left(g_{S_{\theta}} \in d t \mid L\left(S_{\theta}\right)=u\right)=\frac{e^{-\theta t} P_{a}\left(\tau_{u} \in d t\right)}{E_{a}\left(e^{-\theta \tau_{u}}\right)} .
$$

Equations 4.5 and 4.6 imply part (ii) in Theorem 2.1.

\section{Examples}

\subsection{Linear diffusions}

Let $X$ be a regular diffusion on an interval $I \subset \mathbb{R}$ with speed measure $m$. It is well known that $X$ has a jointly continuous density $p(t, x, y)$ with respect to $m$ :

$$
P_{x}\left(X_{t} \in A\right)=\int_{A} p(t, x, y) m(d y) .
$$

The density is symmetric in $x$ and $y$ which implies that for diffusions the dual process is the diffusion itself. Assume that the $X$ has a recurrent point $a$ and that the point $a$ is not an atom of the speed measure $m$. This implies that the inverse local time at $a$ has no drift. With such assumptions the conclusions of the Theorem 2.1 hold with $\hat{X}=X$. Moreover, it is known that

$$
E_{b}\left(e^{-\theta T_{a}}\right)=\frac{r_{\theta}(b, a)}{r_{\theta}(a, a)} \quad \text { and } \quad E_{a}\left(e^{-\theta \tau_{u}}\right)=e^{-\frac{u}{r_{\theta}(a, a)}} .
$$

See Rogers and Williams [25], Sec. 50.

As the first example one can take $X$ to be Brownian motion and $a=0$. All the assumptions are satisfied. It follows that the two processes $\left(B_{t}: 0 \leq t \leq g_{S_{\theta}}\right)$ and $\left(B_{S_{\theta}-t}: 0 \leq t \leq S_{\theta}-g_{S_{\theta}}\right)$ are independent. For the first process we get

$$
E_{0}\left(F\left(B_{t}: 0 \leq t \leq g_{S_{\theta}}\right) \mid L\left(g_{S_{\theta}}\right)=l\right)=\frac{E_{0}\left(F\left(B_{t}: 0 \leq t \leq \tau_{u}\right) e^{-\theta \tau_{u}}\right)}{E_{0}\left(e^{-\theta \tau_{u}}\right)} .
$$


It is well known that

$$
E_{0}\left(e^{-\theta \tau_{l}}\right)=e^{-l \sqrt{2 \theta}} \text { and } \quad E_{b}\left(e^{-\theta T_{0}}\right)=\exp (-|b| \sqrt{2 \theta}) .
$$

where $T_{0}=\inf \left\{t \geq 0: B_{t}=0\right\}$. The first assertion follows from (i) in Theorem 2.1 and the fact that $L_{t} \stackrel{\mathrm{d}}{=}\left|B_{t}\right|$, see e.g. Revuz and Yor [24], p. 289. The hitting time distribution is an elementary consequence of the reflection principle for Brownian motion. The law of the second process, given $B_{S_{\theta}}=a$, is described by

$$
E\left(G\left(B_{S_{\theta}-t}: 0 \leq t \leq S_{\theta}-g_{S_{\theta}}\right) \mid B_{S_{\theta}}=b\right)=\frac{E_{b}\left(F\left(B_{s}: 0 \leq s \leq T_{0}\right) e^{-\theta T_{0}}\right)}{E_{b}\left(e^{-\theta T_{0}}\right)} .
$$

which yields the result first obtained by Biane and Yor in [4]. See Leuridan [17] for an alternative elementary proof and Yen and Yor [28], Ch 9. for an alternative proof.

Since the bridge laws for Brownian motion with drift $B_{t}^{(\mu)}=B_{t}+\mu t$ are exactly the same for all drifts the conditional law of $\left(B_{t}^{(\mu)}: 0 \leq t \leq S_{\theta}\right)$ given $\left\{B_{S_{\theta}}^{(\mu)}=b\right\}$ does not depend on $\mu$. This means that Equations 5.3 and 5.4 hold for Brownian motion with drift with $B$ instead of $B^{(\mu)}$. The only change is that the resolvent density changes to that of Brownian motion with drift

$$
r_{\theta}(a, b)=\frac{1}{\sqrt{2 \theta+\mu^{2}}} e^{\mu(b-a)-|b-a| \sqrt{2 \theta+\mu^{2}}} .
$$

The skew Brownian motion $X^{(\alpha)}$ with parameter $\alpha \in(0,1)$ is constructed by independently flipping the excursions of $\left|B_{t}\right|$ up with probability $\alpha$ and down with probability $1-\alpha$. A pair of dual processes with respect to Lebesgue measure are the processes $X^{(\alpha)}$ and $X^{(1-\alpha)}$. Both processes behave like Brownian motion away from 0 and the distribution of their local time at a fixed point is equal to the distribution of the local time of Brownian motion $X^{(1 / 2)}$. From the known transition densities of $X^{(\alpha)}$, see p. 82 in Revuz and Yor [24], it follows

$$
r_{\theta}(0, b)=(2 \alpha 1(b>0)+2(1-\alpha) 1(b \leq 0)) e^{-|b| \sqrt{2 \theta}} .
$$

The skew Brownian motion satisfies all the assumptions made on the Markov process $X$. Equation 2.7 holds with the same $E\left(e^{-\theta \tau_{u}}\right)$ as in the case of Brownian motion. Equation 2.8 holds with $X$ replaced by standard Brownian motion started at 0 .

Let $X$ be a Bessel process of dimension $\delta \in(0,2)$. Denote $\nu=\delta / 2-1 \in(-1,0)$. It is well known, see Revuz and Yor [24], that 0 is a recurrent point for $X$, satisfying all the assumptions and that the time $X$ spends at 0 has Lebesgue measure 0 . The results of Theorem 2.1 apply. Bessel processes are dual to themselves under the speed measure with density $\xi(d x)=-\frac{x^{2 \nu+1}}{\nu} d x$ on $[0, \infty)$. Let $I_{\nu}(z)$ and $K_{\nu}(z)$ be the modified Bessel functions with index $\nu$. With respect to $\xi$ the transition density of $X$ for $a, b>0$ is given by

$$
p_{t}(a, b)=\frac{1}{t(a b)^{\nu}} e^{-\frac{a^{2}+b^{2}}{2 t}} I_{\nu}\left(\frac{a b}{t}\right) .
$$

Using formula 15.55 in Oberhettinger [19] for $0<a \leq b$ we get that

$$
r_{\theta}(b, a)=\frac{2}{a^{\nu} b^{\nu}} I_{\nu}(a \sqrt{2 \theta}) K_{\nu}(b \sqrt{2 \theta}) .
$$


By Equation 4.2

$$
E_{a}\left(e^{-\theta T_{b}}\right)=\left(\frac{b}{a}\right)^{\nu} \frac{K_{\nu}(a \sqrt{2 \theta})}{K_{\nu}(b \sqrt{2 \theta})}
$$

in accordance with Theorem 3.1 in Kent [15]. By $P_{a}\left(T_{0}<\infty\right)=1$ and the continuity of paths we have $T_{a} \uparrow T_{0}$ as $a \downarrow 0$. Letting $a \rightarrow 0$ and taking into account that $K_{\nu}(z) \sim$ $\frac{\Gamma(-\nu)}{2}(z / 2)^{\nu}$ for $z \rightarrow 0$ we get

$$
E_{b}\left(e^{-\theta T_{0}}\right)=\frac{2^{1+\nu}}{\Gamma(-\nu)}(b \sqrt{2 \theta})^{\nu / 2} K_{\nu}(b \sqrt{2 \theta}) .
$$

By Pitman, Barlow and Yor [1] there is a bicontinuous family of local times $L_{t}^{b}$ of the process $X$ such that

$$
\int_{0}^{t} f\left(X_{s}\right) d s=\frac{1}{2} \int_{0}^{\infty} b^{\delta-1} L_{t}^{b} d b
$$

for bounded measurable functions $f$. With this choice the inverse local time is a stable subordinator of index $-\nu$ with Laplace transform

$$
E_{0}\left(e^{-\theta \tau_{u}}\right)=\exp \left(-u \cdot \frac{2^{1+\nu} \theta^{-\nu} \Gamma(1+\nu)}{\Gamma(-\nu)}\right) .
$$

\subsection{Lévy processes}

For a Lévy process $X$ the Lebesgue measure is invariant and the dual process is $-X$. If the process has continuous densities for $t>0$, is recurrent and spends Lebesgue measure 0 at points the conclusions of Theorem 2.1 can be applied.

An example is provided by symmetric stable processes of index $\alpha \in(1,2)$. These processes are recurrent and by scaling property the inverse local time is a subordinator of index $1-1 / \alpha$. See Bertoin [2], Ch. 8. The independence of $\left(X_{t}: 0 \leq t \leq g_{S_{\theta}}\right)$ and $\left(X_{g_{S_{\theta}}+u}: 0 \leq u \leq S_{\theta}-g_{S_{\theta}}\right)$ and scaling imply that given $g_{1}$ the process $\left(X_{t}: 0 \leq t \leq g_{1}\right)$ is conditionally independent of $\left(X_{g_{1}+u}: 0 \leq u \leq 1-g_{1}\right)$. This means that the two processes

$$
\left(\frac{X_{t g_{1}}}{\sqrt{g_{1}}}: 0 \leq t \leq 1\right) \quad \text { and } \quad\left(\frac{X_{g_{1}+u\left(1-g_{1}\right)}}{\sqrt{1-g_{1}}}: 0 \leq u \leq 1\right)
$$

are independent. Scaling also implies that the inverse local time $\tau_{u}$ is a stable subordinator of index $1-1 / \alpha$ with $E\left(e^{-\theta \tau_{u}}\right)=e^{-u \psi(\theta)}=e^{-c \theta^{1-1 / \alpha}}$ for some constant $c$ depending on the normalization of the local time. From Equation 2.7 we can compute

$$
E\left(e^{-\lambda g_{S_{\theta}}} \mid L\left(S_{\theta}\right)=u\right)=\frac{E\left(e^{-(\lambda+\theta) \tau_{u}}\right)}{E\left(e^{-\theta \tau_{u}}\right)} .
$$

Using the form of $\psi(\theta)$ and unconditioning using Equation 2.6 gives

$$
E\left(e^{-\lambda g_{S_{\theta}}}\right)=\left(\frac{\theta}{\lambda+\theta}\right)^{1-1 / \alpha}
$$

It follows that $g_{S_{\theta}} \sim \Gamma(1-1 / \alpha, \theta)$ and by independence $S_{\theta}-g_{S_{\theta}} \sim \Gamma(1 / \alpha, \theta)$. Using scaling again this gives the arc-sine law $g_{1} \sim \operatorname{Beta}(1-1 / \alpha, 1 / \alpha)$. This result is due to Chaumont [5]. See also Bertoin [2], p. 230. 
For another application let $Y$ be a Lévy process. Assume $P\left(Y_{0}=0\right)=1$ and define $\bar{Y}_{t}=\sup _{s \leq t} Y_{s}$. The reflected process $X=\bar{Y}-Y$ is a Feller process in the right continuous filtration $\mathcal{F}_{t}$ of $X$, see Ch. 6 in Bertoin [2]. Denote by $L$ its local time 0 and let $\tau_{u}=\inf \left\{t: L_{t}>u\right\}$ be the right continuous inverse of $L$. Denote by $\left(e_{u}: u>0\right)$ the excursion process attached to the process $X$. It is in general not possible to reconstruct $\bar{Y}$ from the exursion process of the reflected process $X$. As noted by Greenwood and Pitman in their remark on p. 899 in [10], however, the process of excursions can be extended into a two dimensional Poisson point process such that $\bar{X}$ can be reconstructed. The idea is to add to the excursion at time $u>0$ the jump of the ladder height process $H_{u}=\bar{Y}_{\tau_{u}}$. Denote $J_{u}=H_{u}-H_{u-}$. The resulting point process $\left(\left(e_{u}, J_{u}\right): u>0\right)$ is a Poisson point process in the sense of Itô in the space $U \times(0, \infty)$.

Let $S_{\theta}$ be an exponential random variable with rate $\theta$ independent of $Y$. If the assumptions of Theorem 3.2 are met the following conclusions can be made: (i) The pairs of random variables $\left(g_{S_{\theta}}, \bar{X}_{S_{\theta}}\right)$ and $\left(S_{\theta}-g_{S_{\theta}}, \bar{Y}_{S_{\theta}}-Y_{S_{\theta}}\right)$ are independent. This follows from Theorem 2.1. (ii). The random pair $\left(g_{\theta}, \bar{X}_{\theta}\right)$ is infinitely divisible. To prove this statement first recall a standard result about Lévy processes: if $Z$ is a $d$-dimensional Lévy process and $S_{\theta}$ is an independent exponential random variable, then the random variable $\left(S_{\theta}, Z_{S_{\theta}}\right)$ is infinitely divisible. See Bertoin, [2] p. 162. By Equation 2.8 applied to the reflected process $\tilde{X}$ we find that the law of $\left(g_{S_{\theta}}, \bar{X}_{S_{\theta}}\right)$ given $\left\{\tilde{L}_{\theta}=u\right\}$ is just like the sum of the points of the process $\left(\left(R_{u}, J_{u}\right): u>0\right)$ where $R_{u}$ is the excursion length at local time $u$ and $J_{u}$ is the jump of the ladder height process $H$. This last two-dimensional process is a map of the extended excursion process $\left(\left(e_{u}, J_{u}\right): u>0\right)$ and as such a Poisson point process on $(0, \infty)^{2}$. Sums of Poisson processes are infinitely divisible so it follows that $\left(g_{S_{\theta}}, \bar{X}_{S_{\theta}}\right)$ given $\left\{L_{S_{\theta}}=u\right\}$ is infinitely divisible. But $L_{S_{\theta}}$ is exponentially distributed and infinite divisibility follows. The infinite divisibility of the pair $\left(S_{\theta}-g_{S_{\theta}}, \bar{Y}_{S_{\theta}}-Y_{S_{\theta}}\right)$ follows by duality arguments. See Lemma 9 in Bertoin [2], p. 164.

The assertions about infinite divisibility and independence are true in general without additional assumptions on the reflected process $X$. See Greenwood and Pitman [9] for details.

\section{References}

[1] M. Barlow, J. Pitman and M. Yor, Une extension multidimensionnelle de la loi de l'arc sinus, in: Séminaire de Probabilités, XXIII, Springer, Berlin, volume 1372 of Lecture Notes in Math., pp. 294-314, 1989, doi:10.1007/BFb0083980, http://dx.doi.org/10.1007/ BFb00 083980 .

[2] J. Bertoin, Lévy processes, volume 121 of Cambridge Tracts in Mathematics, Cambridge University Press, 1st edition, 1996.

[3] J. Bertoin, Random fragmentation and coagulation processes, volume 102 of Cambridge Studies in Advanced Mathematics, Cambridge University Press, Cambridge, 2006, doi:10.1017/ CBO9780511617768, http://dx.doi.org/10.1017/CB09780511617768.

[4] P. Biane and M. Yor, Sur la loi des temps locaux browniens pris en un temps exponentiel, in: Séminaire de Probabilités, XXII, Springer, Berlin, volume 1321 of Lecture Notes in Math., pp. 454-466, 1988, doi:10.1007/BFb0084151, http://dx.doi.org/10.1007/ BEb00 84151 .

[5] L. Chaumont, Excursion normalisée, méandre et pont pour les processus de Lévy stables, Bull. Sci. Math. 121 (1997), 377-404. 
[6] P. Fitzsimmons, J. Pitman and M. Yor, Markovian bridges: construction, palm interpretation, and splicing, in: Seminar on Stochastic Processes, 1992, Birkhäuser Boston, Inc., Boston, MA, volume 33 of Progress in Probability, pp. 101-134, 1993, doi:10.1007/978-1-4612-0339-1, http://dx.doi.org/10.1007/978-1-4612-0339-1.

[7] R. K. Getoor and M. J. Sharpe, Last exit decompositions and distributions, Indiana Univ. Math. J. 23 (1973/74), 377-404.

[8] R. K. Getoor and M. J. Sharpe, Excursions of dual processes, Adv. in Math. 45 (1982), 259-309, doi:10.1016/S0001-8708(82)80006-6, http://dx.doi.org/10 . $1016 / \mathrm{S} 0001-8708(82) 80006-6$.

[9] P. Greenwood and J. Pitman, Fluctuation identities for Lévy processes and splitting at the maximum, Adv. in Appl. Probab. 12 (1980), 893-902, doi:10.2307/1426747, http:// dx . doi . org/10.2307/1426747.

[10] P. Greenwood and J. Pitman, Fluctuation identities for random walk by path decomposition at the maximum, Adv. in Appl. Probab. 12 (1980), 291-293, doi:10.2307/1426564, http: //doi.org/10.2307/1426564.

[11] N. Ikeda, M. Nagasawa and K. Sato, A time reversion of Markov processes with killing, Kōdai Math. Sem. Rep. 16 (1964), 88-97.

[12] L. F. James, Bayesian Poisson process partition calculus with an application to Bayesian Lévy moving averages, Ann. Statist. 33 (2005), 1771-1799, doi:10.1214/009053605000000336, http://dx.doi.org/10.1214/009053605000000336.

[13] M. Jeanblanc, J. Pitman and M. Yor, The Feynman-Kac formula and decomposition of Brownian paths, Mat. Appl. Comput. 16 (1997), 27-52.

[14] O. Kallenberg, Splitting at backward times in regenerative sets, Ann. Probab. 9 (1981), 781799, http: / /www. jstor.org/stable/2243738.

[15] J. Kent, Some probabilistic properties of Bessel functions, Ann. Probab. 6 (1978), 760-770.

[16] J. F. C. Kingman, Poisson processes, volume 3 of Oxford Studies in Probability, The Clarendon Press, Oxford University Press, New York, 1993, oxford Science Publications.

[17] C. Leuridan, Une démonstration élémentaire d'une identité de Biane et Yor, in: Séminaire de Probabilités, XXX, Springer, Berlin, volume 1626 of Lecture Notes in Math., pp. 255-260, 1996, doi:10.1007/BFb0094653, http://dx.doi.org/10.1007/BFb0094653.

[18] B. Maisonneuve, Exit systems, Ann. Probab. 3 (1975), 399-411.

[19] F. Oberhettinger and L. Badii, Tables of Laplace transforms, Springer-Verlag, New YorkHeidelberg, 1973.

[20] E. A. Pečerskiı̌ and B. A. Rogozin, The combined distributions of the random variables connected with the fluctuations of a process with independent increments, Teor. Verojatnost. $i$ Primenen. 14 (1969), 431-444.

[21] J. W. Pitman, Lévy systems and path decompositions, in: Seminar on Stochastic Processes, 1981 (Evanston, Ill., 1981), Springer, volume 1 of Progr. Prob. Statist., 1981 pp. 79-110.

[22] A. O. Pittenger and C. T. Shih, Coterminal families and the strong Markov property, Bull. Amer. Math. Soc 78 (1972), 439-443.

[23] D. Ray, Sojourn times of diffusion processes, Illinois J. Math. 7 (1963), 615-630.

[24] D. Revuz and M. Yor, Continuous martingales and Brownian motion, volume 293 of Grundlehren der Mathematischen Wissenschaften [Fundamental Principles of Mathematical Sciences], Springer-Verlag, Berlin, 2nd edition, 1994. 
[25] L. C. G. Rogers and D. Williams, Diffusions, Markov processes, and martingales. Vol. 2: Itô calculus, Wiley Series in Probability and Mathematical Statistics: Probability and Mathematical Statistics, John Wiley \& Sons, Inc., New York, 1987.

[26] P. Salminen, P. Vallois and M. Yor, On the excursion theory for linear diffusions, Jpn. J. Math. 2 (2007), 97-127, doi:10.1007/s11537-007-0662-y, http://dx.doi.org/10.1007/ s11537-007-0662-y.

[27] D. Williams, Path decomposition and continuity of local time for one-dimensional diffusions. I, Proc. London Math. Soc. (3) 28 (1974), 738-768.

[28] J.-Y. Yen and M. Yor, Local times and excursion theory for Brownian motion, volume 2088 of Lecture Notes in Mathematics, Springer, Cham, 2013, doi:10.1007/978-3-319-01270-4, a tale of Wiener and Itô measures, http://dx.doi.org/10.1007/978-3-319-01270-4. 